\title{
First Principles Study of the Properties of a Tetragonal Germanium
}

\author{
Can Zhang, Dong-Dong Pang, Xue-Qian Huang, Han-Yu Xue, Zhen-Long Lv* \\ AND HONG-Ling CUI \\ School of Physics and Engineering, Henan University of Science and Technology, \\ No. 263 Kaiyuan Avenue, Luoyang 471023, China
}

(Received May 22, 2018; in final form September 14, 2018)

\begin{abstract}
Germanium is an important semiconductor having abundant phases of scientific interest. In this work, we studied the structural, electronic, vibrational, elastic and thermal conductivity properties of a tetragonal germanium via first-principles calculations. The results indicate that it is dynamically stable and there is a breathing vibrational mode at its Brillouin zone center. It is a weak metal according to the GGA-based calculation, but an indirect bandgap semiconductor with a gap of $0.24 \mathrm{eV}$ based on the HSE06-functional calculation. According to the calculations performed by the HSE06 functional, both positive and negative hydrostatic pressures can first alter the band gap to be direct and then metallic. The crystal is mechanically stable but anisotropic. Its hardness is predicted to be 8.2 GPa, slightly lower than that of cubic diamond-type Ge. Based on the calculated phonon dispersion curves, its thermal conductivity as a function of temperature is predicted, giving a value of about $13.5 \mathrm{~W} \mathrm{~m}^{-1} \mathrm{~K}^{-1}$ at $300 \mathrm{~K}$.
\end{abstract}

DOI: 10.12693/APhysPolA.134.1163

PACS/topics: 62.20.de, 63.20.D-, 66.25.+g, 71.20.-b

\section{Introduction}

Elements of the group $\mathrm{IV}^{\mathrm{A}}$ in the periodic table have a unique electronic configuration of $s^{2} p^{2}$, which endows them with ability to form different materials with $s p, s p^{2}$ and $s p^{3}$ hybridized bonds. For instance, carbon can form a series of allotropes from zero-dimensional fullerenes [1] to the three-dimensional diamond with various properties, which ignited the interest of researchers, as a result, a number of new phases of carbon were discovered theoretically or experimentally, such as superhard cold compressed carbon [2], full $s p^{2}$ hybridized carbon [3], Ccentered orthorhombic C8 [4], etc.

As a member of the group $\mathrm{IV}^{\mathrm{A}}$ elements, germanium also has many allotropes. It crystallizes in cubic diamond structure at ambient conditions and can adopt the hexagonal diamond structure at certain conditions when being indented or heated from the high-pressure phases [5]. It transforms to $\beta$-Sn-type when pressed up to $11 \mathrm{GPa}[6]$. Further enhancing the pressure to $75-$ $80 \mathrm{GPa}$, it becomes to an orthorhombic Imma phase [7] and then to a hexagonal structure at $81 \mathrm{GPa}$ [8], afterwards, a dense orthorhombic form at about $100 \mathrm{GPa}$ [9]. From then on, it is stable up to $160 \mathrm{GPa}$ [10], but undergoes a transition to a hexagonal close packed phase then [9].

When the pressure is unloaded, $\beta$-Sn-type structure cannot return to the cubic diamond structure but transforms to different metastable phases, such as the simple tetragonal st12 phase [11], rhombohedral r8 phase [12],

*corresponding author; e-mail: 9902883@haust.edu.cn body-centered cubic bc8 structure [13] and hexagonal diamond hd sturcture [14], relying on the unloading process. Bc 8 phase can only be formed under the condition of quick decompression or decompression at low temperature [15], which is unstable and will transform to hd-Ge in a few hours [16]. However, unloading the metallized amorphous Ge often results in bc8-Ge accompanied by a small amount of st12-Ge [16]. Studies reveal that indentation [17] and uniaxial stress [10] can also induce phase transition of Ge. Ge allotropes can also be obtained by other methods: clathrate-type Ge can be synthesized by chemical methods [18]. Allo-Ge and $4 \mathrm{H}-\mathrm{Ge}$ can be formed by delithiating the layered $\mathrm{Li}_{7} \mathrm{Ge}_{12}$ at mild conditions [19]. Ge nanocrystals with the st12 phase can be obtained using amorphous Ge via thermal annealing with the help of certain additives [20].

Most structures of the above allotropes were resolved by relevant experiments and only several were obtained by theoretical prediction [21, 22]. Fujimoto et al. [23] predicted a tetragonal germanium, which belongs to the space group $I_{4} / \mathrm{mmm}$ with eight Ge atoms in the unit cell, but its properties were not well investigated. In this work, we carried out a systematic study on its electronic, vibrational, elastic and thermal conductivity properties by first-principles calculations. The rest of this work is assigned as the following sequence: In Sect. 2, we introduce the computational methods. In Sect. 3, we present and discuss the obtained results, and the conclusions of this work are given in Sect. 4.

\section{Computational details}

In our study, the structure and properties of this tetragonal germanium were studied with the use of the first-principles code VASP [24].GGA-PBE functional [25] 
was used to estimate the exchange-correlation energy of electrons and projector augmented-wave (PAW) method [26] was employed to treat the electron-ion interaction, in which the valence electron configuration of Ge is $4 s^{2} 4 p^{2}$. Wave function was unfolded by a series of plane waves with a cutoff energy of $230 \mathrm{eV}$. The Brillouin zone integration was sampled using a $k$-spacing of $0.4 \AA^{-1}$ based on the Monkhorst-Pack scheme [27]. These parameters ensure that the energy converges within $1 \times$ $10^{-6} \mathrm{eV} /$ atom and the force between ions converges within $0.001 \mathrm{eV} / \AA$. In our test, these parameters lead to a theoretical lattice constant of $5.675 \AA$ for cubic diamondtype $\mathrm{Ge}$, which is very close to the experimental value of $5.646 \AA[28]$. Elastic constants and the phonon-related properties were calculated via finite difference method as implemented in VASP. The phonon spectrum was obtained with the help of the Phonopy code [29].

\section{Results and discussion}

\subsection{Structure and dynamical stability}

Structure of the tetragonal Ge is displayed in Fig. 1. It has a space group of $14 / \mathrm{mmm}$ (No. 139) with eight atoms in the unit cell, thus named T8-Ge for simplicity. The relaxed lattice constants are $a=7.058 \AA$, $c=4.109 \AA$ and the atomic Wyckoff site is $8 \mathrm{~h}$ (0.67907, $0.67907,0.5)$. Four Ge atoms form the Ge4 rings parallel to the $a b$ plane, which are displaced and connected by Ge-Ge bonds along the $c$ axis. There are two kinds of Ge-Ge bonds in T8-Ge: one kind is that in the Ge4 rings, which has a length of $2.526 \AA$ and the other is that along the $c$ axis with a length of $2.497 \AA$. In our calculations, both kinds are longer than that in cubic diamond-type Ge $(2.457 \AA)$. It has a density of $4.71 \mathrm{~g} / \mathrm{cm}^{3}$, smaller than the value $5.28 \mathrm{~g} / \mathrm{cm}^{3}$ of cubic diamond-type Ge. In addition, we also computed their energies, which reveal that the energy of T8-Ge is $87 \mathrm{meV}$ /atom higher than the diamond-type $\mathrm{Ge}$ and about $31 \mathrm{meV} /$ atom higher than the Cco-Ge8 [21].

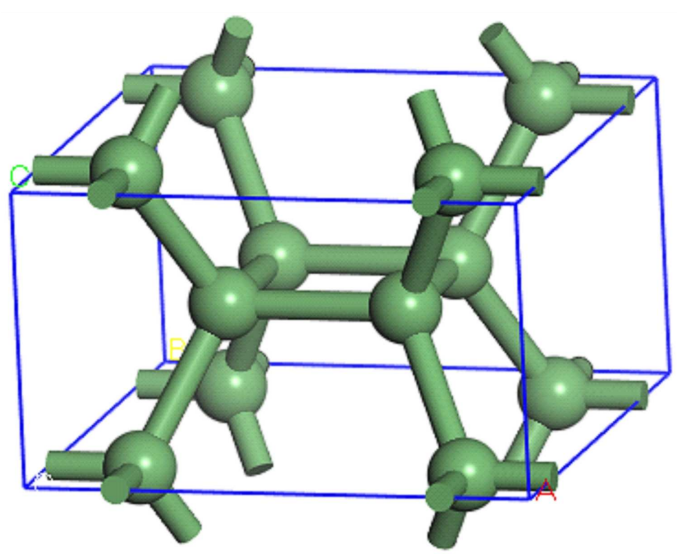

Fig. 1. Crystal structure of the tetragonal germanium (T8-Ge).
To see the dynamical stability of T8-Ge, we computed its phonon dispersion curves and drew it in Fig. 2. It is evident that no imaginary frequencies exist in the Brilloiun zone, which implies that T8-Ge is dynamically stable.

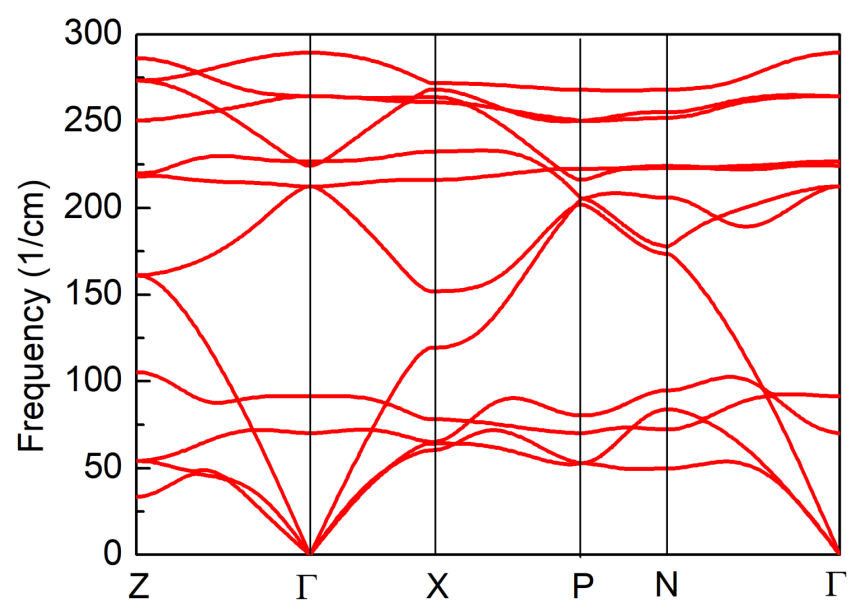

Fig. 2. Calculated phonon dispersion curves of T8-Ge.

We also performed a group factor analysis on the phonons at its Brillouin zone center ( $\Gamma$ point). There are four Ge atoms in the primitive cell of T8-Ge, so there are total twelve vibrational modes: three are acoustic and nine are optical. These optical modes can be resolved into the below irreducible components

$$
\begin{aligned}
\Gamma & =A_{1 g}(R)+B_{1 g}(R)+A_{2 g}(S)+B_{2 g}(R)+B_{2 u}(S) \\
& +E_{g}(R)+E_{u}(I),
\end{aligned}
$$

where $A_{2 g}$ and $B_{2 u}$ are silent modes, $E_{u}$ is a twofold degenerate infrared-active mode and the rest are Ramanactive modes. To give more insight into these modes, we calculated their vibrational frequencies and the corresponding eigenvectors, as shown in Fig. 3.

Those atoms in the two diagonal directions in the $B_{2 u}$ mode move out of phase along the $c$ axis. Those in the Ge4 rings in the $A_{2 g}$ mode vibrate anticlockwise round the $C_{4}$ axis. These two kinds of vibrational manners can-
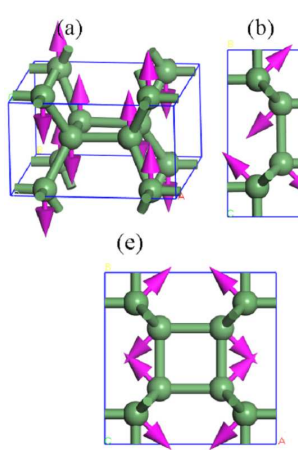
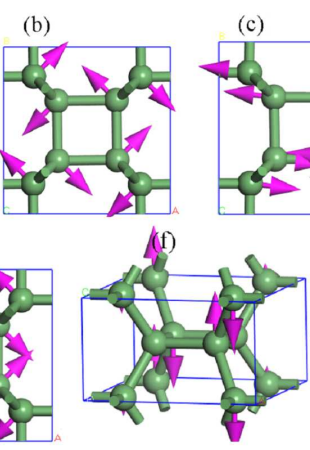

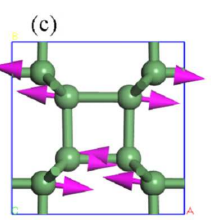

(g)
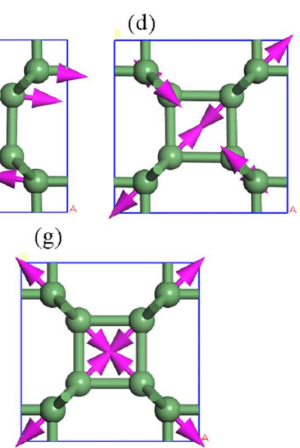

Fig. 3. Calculated vibrational frequencies $\left(\mathrm{cm}^{-1}\right)$ and eigenvectors at the $\Gamma$ point of T8-Ge: (a) $B_{2 u}(70.4)$, (b) $A_{2 g}(91.6)$, (c) $E_{u}(212.5)$, (d) $B_{1 g}(224.5)$, (e) $B_{2 g}$ (227.0), (f) $E_{g}(246.6)$, (g) $A_{1 g}(289.5)$. 
not induce dipole moment and deformation of the electronic cloud, so they are silent. In the $E_{u}$ mode, the top and bottom atoms move out of phase along the $a$ axis, which makes the lengths of these two Ge-Ge bonds unequal and thus induces a weak dipole moment, leading it to be infrared-active. Vibration of the atoms in other modes either results in the same lengths of the Ge-Ge bonds ( $B_{1 g}$ and $A_{1 g}$ modes) in the Ge4 rings or leads to symmetrical distribution of the $\mathrm{Ge}-\mathrm{Ge}$ bonds ( $B_{2 g}$ and $E_{g}$ modes), making the corresponding modes Raman-active. It is interesting to notice that the $A_{1 g}$ mode is a Raman-active breathing mode, which usually has the highest frequency owing to its unique vibrational manner.

\subsection{Electronic properties}

The band structure calculated by the PBE functional is displayed in the left part of Fig. 4. In this case, the Fermi level crosses the valence band at the $Z$ point and meets the conduction band at the $P$ point, which means that T8-Ge is a weak metal in its ground state. Because GGA functional often underestimates band gap of insulators and semiconductors, we recalculated the band structure using the HSE06 functional [30], which is also displayed in the left part of Fig. 4. In this case, the valence band maximum is located at the $Z$ point while the conduction band minimum is at the $P$ point, which indicates that T8-Ge is an indirect band-gap semiconductor although the gap is only $0.24 \mathrm{eV}$. The direct band gap at the $Z$ point is $0.68 \mathrm{eV}$. Bands of T8-Ge seem very dispersive, especially those below $-4 \mathrm{eV}$. From the partial density of states, we can see that the most dispersive bands mainly come from the Ge $4 s$ state. Those between $-4 \mathrm{eV}$ and the Fermi level originate from the Ge $4 p$ state. Those above the Fermi level are formed by the Ge $4 s$ and $4 p$ states. Figure 4 (right part) also shows that the Ge $4 s$ and $4 p$ states overlap with each other in a rather broad energy range, which means hybridization formed between the Ge atoms. The dispersive behavior of the Ge $4 s$ orbital should have a close connection with the hybridization.

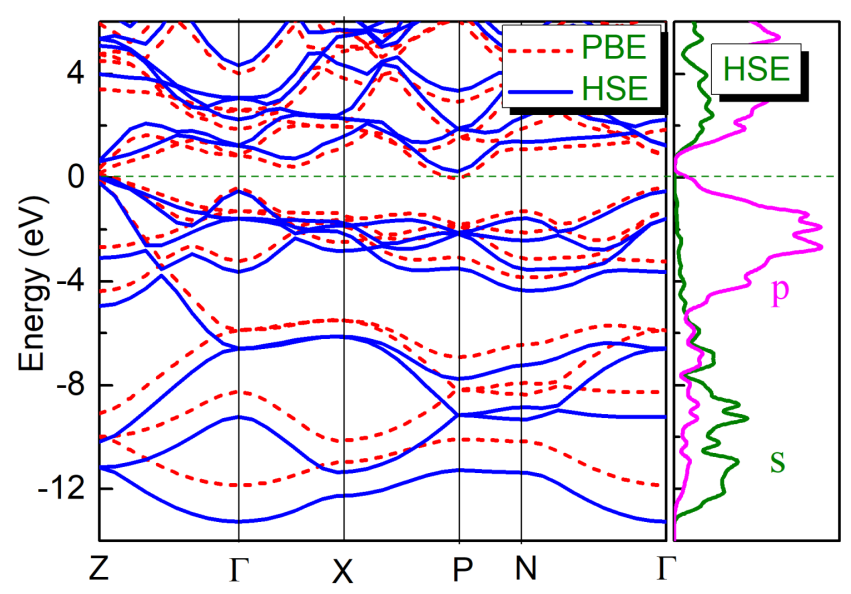

Fig. 4. Calculated band structure (left part) and partial density of states (right part) of T8-Ge.

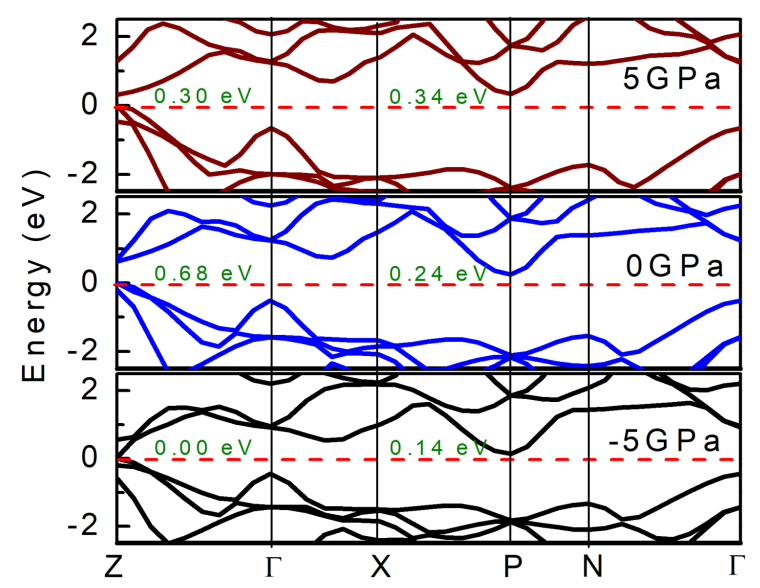

Fig. 5. Band structures of T8-Ge under hydrostatic pressures of $-5,0$, and 5 GPa calculated by the HSE06 functional.

It is expected that pressure or stress will have a noticeable influence on the band structure of T8-Ge since it is very sensitive to the exchange-correlation functional, so we calculated its band structure under the hydrostatic pressures of $-5 \mathrm{GPa}$ and $5 \mathrm{GPa}$ using the HSE06 functional. The results are illustrated in Fig. 5. Under the pressure of $-5 \mathrm{GPa}$, both the valence bands and the conduction bands become narrow and shift towards the Fermi level, making T8-Ge a weak metal via the band crossing at the $Z$ point. Under the pressure of $5 \mathrm{GPa}$, both the valence bands and the conduction bands shift towards the low energy end. One conduction band at the $Z$ point shifts more quickly than the band at the $P$ point, leading T8-Ge to be a direct band gap semiconductor. So, it can be inferred that both positive and negative hydrostatic pressures can first alter the band gap to be direct and then metallic.

\subsection{Elastic properties}

Elastic constants mirror mechanical response of materials under applied strain or stress within their elastic limit, which connect closely with the mechanical stiffness and stability of materials, so they are significant both in theory and experiment.

For a tetragonal crystal, it has six independent elastic constants. If it is mechanically stable, its elastic constants should meet the conditions [31]: $C_{11}>0, C_{33}>0$, $C_{44}>0, C_{66}>0, C_{11}-C_{12}>0, C_{11}+C_{33}-2 C_{13}>0$, $2\left(C_{11}+C_{12}\right)+C_{33}+4 C_{13}>0$. The calculated elastic constants of T8-Ge are $C_{11}=92.2 \mathrm{GPa}, C_{33}=108.8 \mathrm{GPa}$, $C_{44}=31.4 \mathrm{GPa}, C_{66}=19.8 \mathrm{GPa}, C_{12}=31.8 \mathrm{GPa}$ and $C_{13}=34.4 \mathrm{GPa} . C_{33}$ is greater than $C_{11}$, which indicates that the $c$ axis is stiffer than the $a / b$ axis. $C_{44}$ is larger than $C_{66}$, which hints that T8-Ge is more prone to occur shear deformation when the stress is applied on the (100)[010] slip system. $C_{12}$ is close to $C_{13}$, which implies that the $b$ and $c$ axes will have a similar strain when a normal stress is applied along the $a$ axis. 
Large elastic anisotropy can lead to the crack of materials, so it is essential to investigate the elastic anisotropy of T8-Ge. Because the shear related elastic constants $C_{44}$ and $C_{66}$ are smaller than others, we first calculated the shear anisotropic factor of T8-Ge. The factor $A_{1}$ linking the $[011](100)$ and $[010](100)$ shear systems is expressed as $A_{1}=4 C_{44} /\left(C_{11}+C_{33}-2 C_{13}\right)$ while that linking the $[110](001)$ and $[010](001)$ systems is defined as $A_{3}=4 C_{66} /\left(C_{11}+C_{22}-2 C_{12}\right)$ [32]. For materials of shear isotropy, the values of $A_{1}$ and $A_{3}$ equal to one. The calculated results of $A_{1}$ and $A_{3}$ for T8-Ge are 0.95 and 0.66 , respectively, which means that T8-Ge has some shear anisotropy.

Elastic anisotropy can be more vividly exhibited by the directional bulk modulus and Young's modulus. The directional bulk modulus measures whether a material is easy to be compressed or not along different directions under hydrostatic pressure, while the directional Young modulus denotes that property under uniaxial stress. For tetragonal crystals, these two quantities can be derived from the formulae [33]:

$$
\begin{aligned}
& B=\left[\left(S_{11}+S_{12}+S_{13}\right)-\left(S_{11}+S_{12}\right.\right. \\
& \left.\left.\quad-S_{13}-S_{33}\right) l_{3}^{2}\right]^{-1}, \\
& 1 / E=\left(l_{1}^{4}+l_{2}^{4}\right) S_{11}+l_{3}^{4} S_{33}+l_{1}^{2} l_{2}^{2}\left(2 S_{12}+S_{66}\right) \\
& \quad+l_{3}^{2}\left(l-l_{3}^{2}\right)\left(2 S_{13}+S_{44}\right),
\end{aligned}
$$

where $\left(l_{1} l_{2} l_{3}\right)$ is the direction cosine and $S_{i j}$ are the elements of the elastic compliance tensor, the inverse of the elastic tensor.
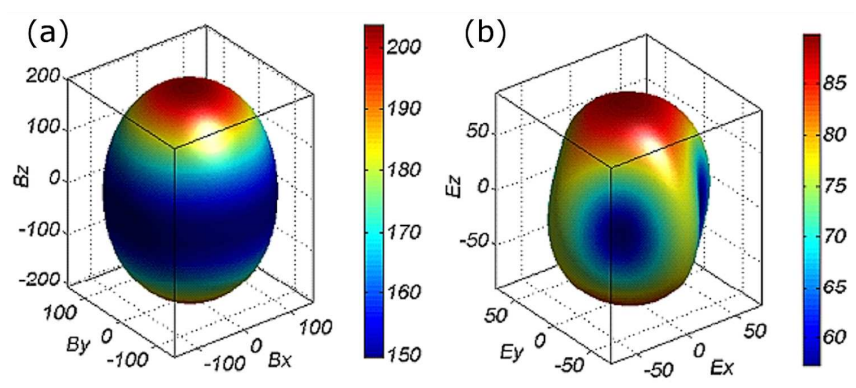

Fig. 6. Calculated directional (a) bulk modulus and (b) Young's modulus of T8-Ge, unit: GPa.

The calculated directional bulk and Young's moduli are given in Fig. 6. It is obvious that T8-Ge is elastically anisotropic because both figures are not spherical. The maximum bulk modulus is 204.1 GPa along the $c$ axis while the minimum is $149.3 \mathrm{GPa}$ in the $a b$ plane, which signifies that the $c$ direction is stiffer than other axes under hydrostatic pressure. The maximum directional Young modulus is $90.1 \mathrm{GPa}$ along the $c$ axis while the minimum is $57.1 \mathrm{GPa}$ in the [110] direction of the (001) plane. The latter is only $63 \%$ as large as the former.

\subsection{Polycrystalline properties and hardness}

For a polycrystalline material, its individual elastic constant is difficult to obtain through experiment, but some comprehensive quantities, such as bulk modulus $B$, shear modulus $G$, and Poisson's ratio $v$ can be directly measured. These values can also be calculated with the use of the formulae given in Ref. [31]. The bulk and shear moduli calculated using Voigt's method are 54.9 and 29.4 GPa, respectively. These obtained using Reuss' method are 54.5 and $28.2 \mathrm{GPa}$, respectively. They finally give the bulk modulus $54.7 \mathrm{GPa}$ and the shear modulus 28.8 GPa for T8-Ge. The bulk modulus is about twice as large as the shear modulus, which indicates that T8-Ge is more resistant to volume change than shape deformation, so the main factor deciding the mechanical stability of $\mathrm{T} 8 \mathrm{-Ge}$ is the shear deformation.

Pugh [34] proposed that the $B / G$ ratio can be used to predict brittleness and ductility of materials: a value beyond 1.75 for ductile materials while below it for brittle materials. The $B / G$ ratio of $\mathrm{T} 8-\mathrm{Ge}$ is 1.90 , which means that it is ductile. The calculated transverse acoustic velocity $\left(V_{t}\right)$ of $\mathrm{T} 8-\mathrm{Ge}$ is $2466.1 \mathrm{~m} / \mathrm{s}$ and the longitudinal acoustic velocity $\left(V_{l}\right)$ is $4434.3 \mathrm{~m} / \mathrm{s}$. The average acoustic velocity $\left(V_{m}\right)$ can be deduced to be $2746.4 \mathrm{~m} / \mathrm{s}$, which ultimately gives a value of $278.0 \mathrm{~K}$ for the Debye temperature of T8-Ge. It is considerably lower than the value $400 \mathrm{~K}$ of diamond-type Ge [35]. It is always thought that a high Debye temperature means a high thermal conductivity [36], so T8-Ge should not be a good thermal conductor in this sense.

Jiang et al. [37] put forward the empirical formulae $B=10.38 \mathrm{H}_{V}$ and $G=6.78 \mathrm{H}_{V}$ to evaluate the hardness of covalent materials. Based on these two formulae, we got the values 5.27 (6.4) $\mathrm{GPa}$ and 4.25 (5.9) $\mathrm{GPa}$ as the hardness of T8-Ge (diamond-type Ge). Chen et al. [38] also proposed an empirical formula $H_{V}=2\left(k^{2} G\right)^{0.585}-3$, where $k=G / B$, to estimate the hardness of materials. Putting the obtained moduli into it, we got a value of 3.74 (6.5) $\mathrm{GPa}$ as the hardness of T8-Ge (diamond-type Ge). These values are considerably smaller than the experi-

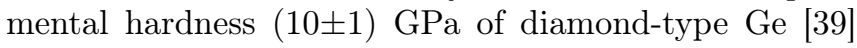
measured at room temperature.

From a microcosmic view, hardness is thought to be decided by three factors: electronic density per volume of the crystal, ionicity and strength of bonds within the crystal [40]. Here we use the method proposed by Simůnek [41] to calculate the hardness of T8-Ge:

$$
\begin{aligned}
& H=\frac{C}{V} n\left(\prod_{i, j=1}^{n} N_{i j} S_{i j}\right)^{1 / n} \exp \left(-\sigma f_{e}\right), \\
& f_{e}=1-\left[k\left(\prod_{i=1}^{k} e_{i}\right)^{1 / k} / \sum_{i=1}^{k} e_{i}\right]^{2}, \\
& S_{i j}=\sqrt{e_{i} e_{j}} /\left(n_{i} n_{j} d_{i j}\right),
\end{aligned}
$$

where $e_{i}=Z_{i} / R_{i}$ is the reference energy of the atom $i$, here $Z_{i}$ denotes the number of the valence electrons of the atom $i$ and $R_{i}$ is its radius; $S_{i j}$ is the strength of the bond formed by the atoms $i$ and $j$ while $N_{i j}$ is the number of the corresponding bonds in the cell; $\exp \left(-\sigma f_{e}\right)$ 
is used to measure the effect caused by the difference of reference energies of the bonded atoms; $d_{i j}$ is the length of the bond formed by the atoms $i$ and $j ; n_{i}\left(n_{j}\right)$ is the coordination number of the atom $i(j) ; k$ and $n$ are the types of atoms and bonds, respectively.

As a test, we first computed the hardness of the diamond-type Ge. In it, both the number of $\mathrm{Ge}-\mathrm{Ge}$ bonds and the coordination number of each Ge are four. The radius of Ge is taken as $1.37 \AA$ from the textbook [42] and the number of valence electrons is four. After relaxation, the length of $\mathrm{Ge}-\mathrm{Ge}$ bonds is $2.457 \AA$ and the cell volume is $45.68 \AA^{3}$. These parameters finally give a value of $9.4 \mathrm{GPa}$ as the hardness of diamond-type $\mathrm{Ge}$, which agrees well with the experimental value $(10 \pm 1) \mathrm{GPa}$ [39]. As for T8-Ge, we gained a value of about $8.2 \mathrm{GPa}$ as its hardness, which is slightly lower than that of the diamond-type Ge. We can see that the pure empirical methods used above cannot give accurate hardnesses as the more meaningful model proposed by Šimůnek.

\subsection{Thermal conductivity}

We know that heat is conducted mainly by the acoustic phonons. The calculated phonon spectrum of T8-Ge reveals that some optical modes tangle with the acoustic ones, which will influence the thermal conductivity of T8-Ge. Following, we gave a theoretical estimation employing the method recently put forward by Garrity [43]:

$$
\begin{aligned}
& k_{l}(T)=\frac{0.849 \times 3 \sqrt[3]{4}}{20 \pi^{3}\left(1-0.514 \gamma^{-1}+0.228 \gamma^{-2}\right)} \\
& \times\left(\frac{k_{\mathrm{B}} \Theta_{\mathrm{D}}}{\hbar}\right)^{2} \frac{k_{\mathrm{B}} \bar{M} V^{1 / 3}}{\hbar \gamma^{2}} \frac{\Theta_{\mathrm{D}}}{T},
\end{aligned}
$$

where $\bar{M}$ is the averaged atomic masses, $\Theta_{\mathrm{D}}$ is the Debye temperature and $\gamma$ is the Grüneisen parameter. The latter two quantities can be obtained from the phonon dispersion spectrum utilizing the formulae

$$
\begin{aligned}
& \Theta_{D}=n^{-1 / 3} \sqrt{\frac{5 \hbar^{2}}{3 k_{\mathrm{B}}^{2}} \frac{\int_{0}^{\infty} \omega^{2} g(\omega) \mathrm{d} \omega}{\int_{0}^{\infty} g(\omega) \mathrm{d} \omega}} \\
& \gamma^{2}=\frac{\sum_{i} \int \frac{\mathrm{d} q}{8 \pi^{3}} \gamma_{i q}^{2} C_{i q}}{\sum_{i} \int \frac{\mathrm{d} q}{8 \pi^{3}} C_{i q}}
\end{aligned}
$$

where $n$ counts the number of atoms in cell, $\omega$ is the vibrational frequency of phonon, $g(\omega)$ represents the total density of states of the phonon, $\gamma_{i q}$ is the mode Grüneisen parameter and $C_{i q}$ is its specific heat, which can be derived from the computed vibrational frequency of mode $i$ using the definitions:

$$
\begin{aligned}
\gamma_{i q} & =-\frac{V}{\omega_{i q}} \frac{\partial \omega_{i q}}{\partial V} \\
C_{i q} & =k_{\mathrm{B}}\left(\frac{\hbar \omega_{i q}}{k_{\mathrm{B}} T}\right)^{2} \frac{\mathrm{e}^{-\hbar \omega_{i q} / k_{\mathrm{B}} T}}{\left(\mathrm{e}^{-\hbar \omega_{i q} / k_{\mathrm{B}} T}-1\right)^{2}} .
\end{aligned}
$$

The melting temperature of the diamond-type Ge is reported to be $1200 \mathrm{~K}$ [44]. The melting temperature of T8-Ge should be lower than it. For simplicity, here we calculated its thermal conductivity in the temperature range from 200 to $1200 \mathrm{~K}$, where the Umklapp scattering is thought to be the primary mechanism of phonon scattering. The estimated thermal conductivity as a function of temperature is drawn in Fig. 7 (starred blue line).

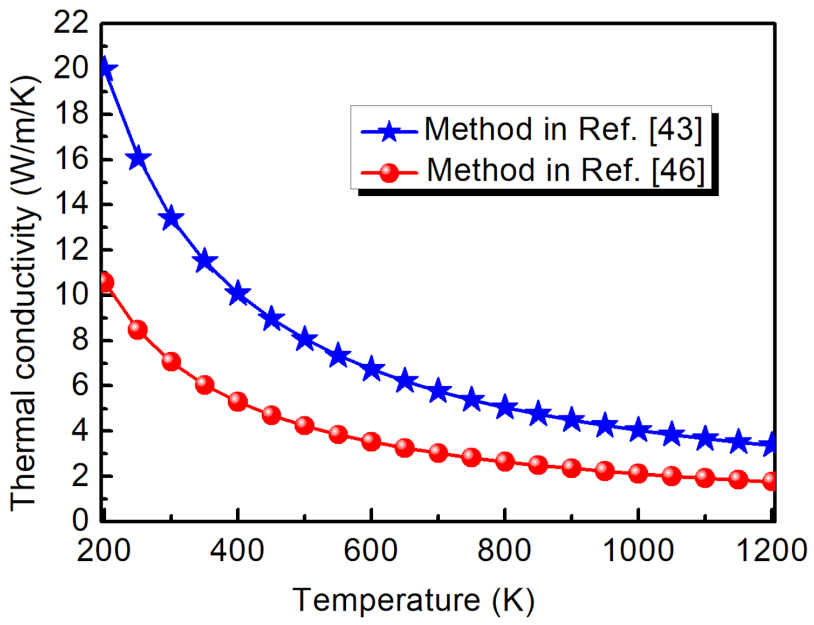

Fig. 7. Calculated thermal conductivity of T8-Ge as a function of temperature.

It is found that the thermal conductivity of T8-Ge decreases as the temperature rises. The reason for it is that the coupling between phonons changes weak when temperature rises, which causes the phonon mean-free path shorten to the average atomic distance [45]. At $300 \mathrm{~K}$, our simulation gives a value of $13.5 \mathrm{~W} \mathrm{~m}^{-1} \mathrm{~K}^{-1}$ as the thermal conductivity of T8-Ge, which is far below the experimental value $60 \mathrm{~W} \mathrm{~m}^{-1} \mathrm{~K}^{-1}$ of the diamond-type Ge at the same temperature [35]. One reason may be that the twist of the optical and the heat-carrying acoustic phonons in T8-Ge is strong, so the heat scattering in it is serious than that in the diamond-type Ge. Besides, we found that the Debye temperature derived from Eq. (8) is about $223.4 \mathrm{~K}$, which is lower than the value $278.0 \mathrm{~K}$ obtained from the elastic constants due to the different computational methods.

Using the above method to calculate thermal conductivity needs to calculate the phonon dispersion spectrum, which is usually time-consuming. A simple method given by Slack [46] is often used to evaluate thermal conductivity of materials, which is formulated as: $k=$ $3.1 \times 10^{-6} \frac{\bar{M} \Theta_{\mathrm{D}}^{3} \delta}{\gamma_{\alpha}^{2} n^{2 / 3} T}$, where $\Theta_{\mathrm{D}}$ is the Debye temperature obtained from elastic constants, $\delta^{3}$ is the volume per atom, $\gamma_{\alpha}$ is the Grüneisen parameter of acoustic modes, which can be gained from the Poisson ratio via [47]: $\gamma_{\alpha}=\frac{3(1+\nu)}{2(2-3 \nu)}$. The thermal conductivity curve obtained using this method is also drawn in Fig. 7 (red line with balls). It is evident that the thermal conductivity at 
each temperature calculated based on this method is lower than that obtained from the former. We also used both methods to calculate the thermal conductivity of the diamond-type Ge and found that the curve obtained from the former method is beyond the experimental one while that obtained from the latter is underneath it, so we infer that the thermal conductivity curve of T8-Ge should lie between these two curves shown in Fig. 7.

\section{Conclusions}

In this work, we systematically studied the structural, electronic, vibrational, elastic, and thermal conductivity properties of a tetragonal germanium by first-principles calculations. The results indicate that it is dynamically stable. Group factor analysis was performed and the vibrational frequencies and eigenvectors at the Brillouin zone center were calculated. GGA-based calculation indicates that T8-Ge is a weak metal, but HSE06-based calculation implies that it is an indirect band-gap semiconductor with a gap of $0.24 \mathrm{eV}$. Using the HSE06 functional, we found that both positive and negative hydrostatic pressures can first alter the band gap to be direct and then metallic. Obtained elastic constants and related properties imply that T8-Ge is mechanically stable but anisotropic. Different hardness models were used to estimate its hardness and $8.2 \mathrm{GPa}$ should be the most reliable one. Its thermal conductivity as a function of temperature was computed on the basis of the calculated phonon dispersion curves. The predicted value at $300 \mathrm{~K}$ is about $13.5 \mathrm{~W} \mathrm{~m}^{-1} \mathrm{~K}^{-1}$, which is higher than the value $7 \mathrm{~W} \mathrm{~m}^{-1} \mathrm{~K}^{-1}$ obtained using the sample Slack model.

\section{Acknowledgments}

This work was supported by the Student Research Training Program of School of Physics and Engineering (Grant No. 201701) of Henan University of Science and Technology, and the Key Scientific Research Projects of Henan Colleges and Universities (Grant No. 16A140008).

\section{References}

[1] H.W. Kroto, J.R. Heath, S.C. O'Brien, R.F. Curl, R.E. Smalley, Nature 318, 162 (1985).

[2] W.L. Mao, H.K. Mao, P.J. Eng, T.P. Trainor, M. Newville, C.C. Kao, D.L. Heinz, J. Shu, Y. Meng, R.J. Hemley, Science 302, 425 (2003).

[3] J.T. Wang, C. Chen, Y. Kawazoe, Sci. Rep. 3, 3077 (2013).

[4] Z.S. Zhao, B. Xu, X.F. Zhou, L.M. Wang, B. Wen, J.L. He, Z.Y. Liu, H.T. Wang, Y.J. Tian, Phys. Rev. Lett. 107, 215502 (2011).

[5] S. Ruffell, B. Haberl, S. Koenig, J.E. Bradby, J.S. Williams, J. Appl. Phys. 105, 093513 (2009).

[6] R.J. Nelmes, M.I. McMahon, Semicond. Semimet. 54, 145 (1998).

[7] R.J. Nelmes, H. Liu, S.A. Belmonte, J.S. Loveday, M.I. McMahon, D.R. Allan, D. Hausermann, M. Hanfland, Phys. Rev. B 53, R2907 (1996).
[8] Y.K. Vohra, K.E. Brister, S. Desgreniers, A.L. Ruoff, K.J. Chang, M.L. Cohen, Phys. Rev. Lett. 56, 1944 (1986).

[9] K. Takemura, U. Schwarz, K. Syassen, M. Hanfland, N.E. Christensen, D.L. Novikov, I. Loa, Phys. Rev. B 62, R10603 (2000).

[10] M. Durandurdu, Phys. Rev. B 71, 054112 (2005).

[11] F.P. Bundy, J.S. Kasper, Science 139, 340 (1963).

[12] R.O. Piltz, J.R. Maclean, S.J. Clark, G.J. Ackland, P.D. Hatton, J. Crain, Phys. Rev. B 52, 4072 (1995).

[13] C.H. Bates, F. Dachille, R. Roy, Science 147, 860 (1965).

[14] J.S. Kasper, J.R.H. Wentorf, Science 197, 599 (1977).

[15] A.G. Lyapin, V.V. Brazhkin, S.V. Popova, A.V. Sapeliki, Phys. Status Solidi B 198, 481 (1996).

[16] R.J. Nelmes, M.I. McMahon, N.G. Wright, D.R. Allan, J.S. Loveday, Phys. Rev. B 48, 9883 (1993).

[17] G. Patriarche, E.L. Bourhis, M.M.O. Khayyat, M.M. Chaudhri, J. Appl. Phys. 96, 1464 (2004).

[18] M. Beekman, G.S. Nolas, J. Mater. Chem. 18, 842 (2008).

[19] A. Grüttner, R. Nesper, H.G. Schnering, Angew. Chem. Int. Ed. 21, 912 (1982).

[20] S.J. Kim, O.K. Quy, L.S. Chang, E.A. Stach, C.A. Handwerker, A. Wei, J. Mater. Chem. 20, 331 (2010).

[21] J. Zhai, D. Yu, K. Luo, Q. Wang, Z. Zhao, J. He, Y. Tian, J. Phys. Condens. Matter 24, 405803 (2012).

[22] Z. Zhao, F. Tian, X. Dong, Q. Li, Q. Wang, H. Wang, X. Zhong, B. Xu, D. Yu, J. He, H.T. Wang, Y. Ma, Y. Tian, J. Am. Chem. Soc. 134, 12362 (2012).

[23] Y. Fujimoto, T. Koretsune, S. Saito, T. Miyake, A. Oshiyama, New J. Phys. 10, 083001 (2008).

[24] G. Kresse, J. Furthmüller, Phys. Rev. B 54, 11169 (1996).

[25] J.P. Perdew, K. Burke, M. Ernzerhof, Phys. Rev. Lett. 77, 3865 (1996).

[26] G. Kresse, D. Joubert, Phys. Rev. B 59, 1758 (1999).

[27] H.J. Monkhorst, J.D. Pack, Phys. Rev. B 13, 5188 (1976).

[28] A.S. Cooper, Acta Crystallogr. 15, 578 (1962).

[29] A. Togo, F. Oba, I. Tanaka, Phys. Rev. B 78, 134106 (2008).

[30] J. Heyd, G.E. Scuseria, M. Ernzerhof, J. Chem. Phys. 118, 8207 (2003).

[31] Z.L. Lv, H.L. Cui, H. Wang, X.H. Li, G.F. Ji, Diam. Relat. Mater. 71, 73 (2017).

[32] P. Ravindran, L. Fast, P.A. Korzhavyi, B. Johansson, J. Wills, O. Eriksson, J. Appl. Phys. 84, 4891 (1998).

[33] Z.L. Lv, Y. Cheng, X.R. Chen, G.F. Ji, Comput. Mater. Sci. 77, 114 (2013).

[34] S.F. Pugh, Philos. Mag. 45, 833 (1954).

[35] G.A. Slack, C. Glassbrenner, Phys. Rev. 120, 782 (1960).

[36] A. Bouhemadou, R. Khenata, S. Binomran, Physica B 406, 2851 (2011).

[37] X. Jiang, J.J. Zhao, X. Jiang, Comput. Mater. Sci. 50, 2287 (2011). 
[38] X.Q. Chen, H. Niu, D. Li, Y. Li, Intermetallics 19, 1275 (2011).

[39] L.J. Vandeperre, F. Giuliani, S.J. Lloyd, W.J. Clegg, Acta Mater. 55, 6307 (2007).

[40] F. Gao, R. Xu, K. Liu, Phys. Rev. B 71, 052103 (2005).

[41] A. Šimůnek, J. Vackář, Phys. Rev. Lett. 96, 085501 (2006).

[42] C. Kittel, Introduction to Solid State Physics, Wiley, New York 2005, p. 71.
[43] K.F. Garrity, Phys. Rev. B 94, 045122 (2016).

[44] C.J. Glassbrenner, G.A. Slack, Phys. Rev. 134, A1058 (1964).

[45] D.R. Clarke, Surf. Coat. Technol. 163, 67 (2003).

[46] G.A. Slack, J. Phys. Chem. Solids 34, 321 (1973).

[47] B.D. Sanditov, S.B. Tsydypov, D.S. Sanditov, Acoust. Phys. 53, 594 (2007). 